\title{
SEMICLASSICAL CALCULATION OF FISSION BARRIERS WITH SKYRME FORCES
}

\author{
C. GUET ', H.-B. HAKANSSON and M. BRACK \\ Institute of Theoretical Physics, University of Regensburg, D-8400 Regensburg, West Germany
}

Received 21 August 1980

\begin{abstract}
We present semiclassically calculated fission barriers for ${ }^{240} \mathrm{Pu}$ starting from effective nuclear interactions of the Skyrme type. We show that at least one of the existing Skyrme parametrisations leads to a reasonably low fission barrier.
\end{abstract}

The recent development of simple phenomenological, yet realistic effective nuclear interactions $[1,2]$ has made it possible to calculate static fission barriers of heavy nuclei with the constrained Hartree-Fock (CHF) method $[3,4]$. However, the barriers obtained in this way for ${ }^{240} \mathrm{Pu}$ are higher than the experimental ones by almost a factor 2 even after a careful estimation of several possible uncertainties $[4,5]$. This presents a challenging puzzle to the theoretician, since the same forces used with the HF method lead to an agreement with many ground state properties of spherical and deformed nuclei throughout the mass table (see e.g. the review article ref. [6]).

Before raising serious doubts about the validity of the CHF method used at large deformations, it is necessary to investigate whether it is not possible to refit the effective force parameters so as to get reasonable fission barriers without spoiling the good agreement obtained for the ground state properties. In fact, there is still a lot of freedom left at least in the case of the Skyrme type forces, for which numerous parametrisations exist which all give comparable results for spherical nuclei (see e.g. refs. $[1,7]$ ). Unfortunately, the CHF calculations for heavy deformed nuclei still re. quire enormously long computer times which make systematical investigations of fission barriers practically impossible.

\footnotetext{
Work partially supported by the Deutsche Forschungsgemeinschaft DFG grant no. Br 722/1.

1 Permanent address: DRF/CPN, CEN, F-38041 Grenoble, France.
}

In this note we want to present semiclassical calculations of fission barriers for a series of Skyrme forces. Our method is essentially based on the energy density formalism. With the help of the semiclassical functionals $\tau[\rho]$ and $J[\rho]$ obtained from the extended ThomasFermi (ETF) model (see, e.g. refs. [8,9]), we express the kinetic energy density $\tau(r)$ and the spin-orbit density $J(r)$ (see ref. [1]) for each kind of nucleons by the corresponding nucleon densities. Using the Skyrme force, the parameters of which we take from spherical HF calculations $[1,7]$, the total binding energy of the nucleus is then given uniquely in terms of the neutron and proton densities $\rho_{\mathrm{n}}(r), \rho_{\mathrm{p}}(r)$ and their gradients. To obtain deformation energy curves, we parametrise the densities for suitably deformed nuclear shapes and vary their parameters so as to minimise the total energy under a given external constraint (e.g. the quadrupole moment or the length of the nucleus). Some preliminary results of such calculations have already been presented recently [10].

This method allows us, as we show here for $240 \mathrm{Pu}$, to obtain in a very economical way the average fission barriers of different Skyrme type forces and to compare them to the empirically known liquid drop barrier. Shell effects, which are not contained in the present treatment, can be included perturbatively as demonstrated by detailed numerical investigations [11]. This will be done in a forthcoming publication.

We use the full ETF functionals $\tau[\rho]$ and $J[\rho]$ given in ref. [9], including all effective mass and spin-orbit contributions and gradients of the density $\rho$ up to fourth order. (It is worth mentioning that under the 
spatial integration required for the total energy, the number of terms reduces greatly and actually only first and second derivatives of $\rho(r)$ are needed.) The kinetic energy density functional $\tau[\rho]$ has recently been tested for different deformed local potentials $[8,12]$ and found to reproduce the total average $\mathrm{ki}$ netic energies with considerable accuracy. We can conclude from these results that the error in relative deformation energies stemming from the use of this functional should be less than $\approx 1-2 \mathrm{MeV}$.

The basic input in our calculations are the nucleon densities which must be suitably parametrised in order to describe deformed nuclear shapes with a diffuse surface. For that we have adopted a technique [13] which has proved successful for defining a deformed average nuclear potential in extended shell-correction calculations [14]. In cylindrical coordinates $z, r$ (keeping axial symmetry), our densities are defined by

$\rho_{\mathrm{q}}(r, z)=\rho_{0 \mathrm{q}} /\left\{1+\exp \left[l_{\mathrm{q}}(r, z) / a_{\mathrm{q}}\right]\right\} \quad(\mathrm{q}=\mathrm{n}, \mathrm{p})$,

where the central densities $\rho_{0 \mathrm{q}}$ and the surface diffuseness parameters $a_{\mathrm{q}}$ are allowed to be different for neutrons and protons. The length variable $l_{\mathrm{q}}(r, z)$ is defined by

$l_{\mathrm{q}}(r, z)=\Pi_{\mathrm{q}}(r, z ; \operatorname{def}) /\left|\nabla_{r, z} \Pi_{\mathrm{q}}(r, z ; \operatorname{def})\right|$,

and depends on the deformation through the shape function $\Pi_{\mathrm{q}}(r, z ;$ def $)$. The deformed nuclear surface is defined by $\Pi_{\mathrm{q}}(r, z ;$ def $)=0$ in terms of some shape parameters denoted at the moment by "def" and specified below. The length variable $l_{\mathrm{q}}$ measures thus the distance from the nuclear surface in the direction of its gradient which is held constant due to the definition eq. (2) (see refs. [13,14] for details).

We have used the $\{c, h\}$ parametrisation of ref. [14] which is well suited to describe fission shapes up to the second barrier region of actinide nuclei. It becomes poor for very large deformations $(c \approx 2)$ when the corresponding liquid drop comes close to scission. We therefore used at large deformations a three-parameter parametrisation $\{s, t, v\}$ which was developed in ref. [10] and consists of matching two ellipsoids (for the "ends" of the nucleus) with a sixth order polynomial (for the neck region). The parameters $\{s, t, v\}$ can be related uniquely to the total length $L$ of the nucleus in $z$ (symmetry axis) direction, the neck radius (at $z$ $=0$ ) and the maximum radius in $r$ direction (see ref. [10] for the details).
The direct part of the Coulomb energy was calculated for a sharp-surface liquid drop with an equivalent radius (determined by fixing the proton number). As shown by Myers and Swiatecki [15], this gives the correct deformation dependence up to order $\left(a_{\mathrm{p}} / R_{\mathrm{p}}\right)^{2}$ in the leptodermous expansion which can be assumed to hold up to the saddle point deformations considered here. We checked that e.g. in the ${ }^{208} \mathrm{~Pb}$ ground state, inclusion of the term $\left(a_{\mathrm{p}} / R_{\mathrm{p}}\right)^{2}$ reproduces to within $\approx 1 \mathrm{MeV}$ the exact Coulomb energy obtained from the diffuse proton distribution $\rho_{\mathrm{p}}(r)$. The exchange Coulomb energy was included in the Slater approximation as is usual also in Skyrme HF calculations $[1,3,11]$.

In fig. 1 we show the deformation energy obtained for ${ }^{240} \mathrm{Pu}$ with the Skyrme force S III, plotted against the total length $L$ of the nucleus (taken as the distance between the two half-density points along the $z$ axis). For each value of $L$, the total energy has been minimised with respect to $a_{\mathrm{n}}, a_{\mathrm{p}}, \rho_{0 \mathrm{n}}$ and $\rho_{0 \mathrm{p}}$ and to the one (or two) remaining shape parameter(s). (The halfdensity radii $R_{\mathrm{n}}$ and $R_{\mathrm{p}}$ contained in $\Pi_{\mathrm{q}}$ are fixed by particle number conservation.) The upper curve is obtained with the $\{c, h\}$ parametrisation, the dashed curve with the $\{s, t, v\}$ parametrisation. The two curves are identical up to $L \approx 22 \mathrm{fm}$. For larger deformations, the $\{s, t, v\}$ shapes lead to lower energies than the $\{c, h\}$ shapes. The dashed-dotted curve $\left(\widetilde{E}_{\mathrm{CHF}}\right)$ is the result of a CHF calculation, obtained by constraining the

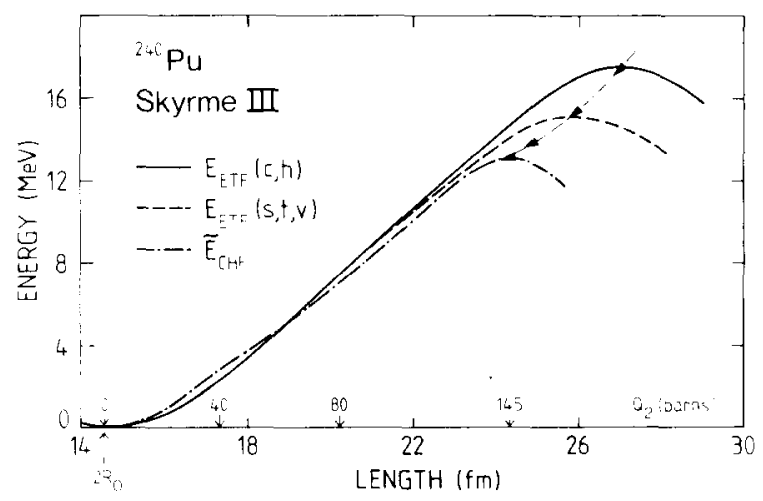

Fig. 1. Deformation energies (in MeV) obtained with the Skyrme force S III for ${ }^{240} \mathrm{Pu}$ using two different shape parametrisations, plotted versus the total length $L$ (in $\mathrm{fm}$ ) of the nucleus. ( $R_{0}$ is the average of the spherical proton and neutron half -density radii $R_{\mathrm{n}}$ and $R_{\mathrm{p}}$ ) The averaged HF result $\left(E_{\mathrm{CHF}}\right)$ from ref. [11] is shown as a function of the quadrupole moment $Q_{2}$ also indicated along the abscissa. 
mass quadrupole moment $Q_{2}$, Strutinsky averaged in order to eliminate the shell effects [11]. (The weak bump in $\widetilde{E}_{\mathrm{CHF}}$ around $Q_{2} \approx 40 \mathrm{~b}$ is due to a lack of convergence in the selfconsistent iteration, as explained in ref. [11].) We get an excellent agreement between the semiclassical and the averaged CHF curves (better than $1 \mathrm{MeV}$ ) up to $L \approx 23 \mathrm{fm}$. A priori, the constraints on $L$ and $Q_{2}$ need not lead to the same fission path between the extremal points; the corresponding difference in energy should, however, be less than $\approx 1 \mathrm{MeV}$. At the saddle point, the semiclassical energy $E_{\mathrm{ETF}}(s, t, v)$ is still $\approx 2 \mathrm{MeV}$ higher than the average CHF energy.

Comparing with the preliminary results in ref. [10], the barrier height has decreased by $\approx 3 \mathrm{MeV}$ by allowing different parameters for protons and neutrons. Most of this gain $(\approx 2 \mathrm{MeV})$ is brought about by the different values of the diffuseness parameters $a_{\mathrm{n}}$ and $a_{\mathrm{p}}$. While improving the parametrisation, the saddle point moved along the dashed line indicated with arrows, which allows us to extrapolate that by optimising the density shapes one might actually come very close to the CHF saddle point. The remaining difference at $L \gtrsim 23 \mathrm{fm}$ can thus be attributed to the restriction of the variational space of our nuclear densities. It should be noted that the present test case is rather pathological since with the force S III the barrier is far too high $(\approx 13 \mathrm{MeV}$ instead of $\approx 4 \mathrm{MeV}$, see also fig. 2 below) and consequently the saddle point is at unrealistically large deformations $\left(Q_{2} \approx 145 \mathrm{~b}\right.$ instead of $\approx 100 \mathrm{~b}$ ). For forces which lead to realistic barrier heights, we can therefore expect to be at deformations where the semiclassical results are well converged and agree with the $\mathrm{HF}$ result to within less than $1 \mathrm{MeV}$. Considering the simplicity of our approach, this result can be considered as very satisfactory.

In fig. 2 we present the results obtained for the ${ }^{240} \mathrm{Pu}$ fission barriers for 5 different Skyrme force parameter sets. The $\{s, t, v\}$ shapes were used above $L \approx 22 \mathrm{fm}$. The forces S III to S VI (see Beiner et al. [1]) lead to very similar barrier heights. The force SkM [7] which distinguishes itself from the others mainly by its lower, more realistic incompressibility $(K \approx 220$ $\mathrm{MeV}$ ), gives an appreciably lower fission barrier of $\approx 10 \mathrm{MeV}$. We also show the empirical LDM barrier calculated with the parameters of ref. [15] along the $\{c, h\}$ fission path $(h \approx 0$, see ref. [14]). Our results are qualitatively corroborated by a recent non-selfconsistent microscopic study of fission barriers [16], al-

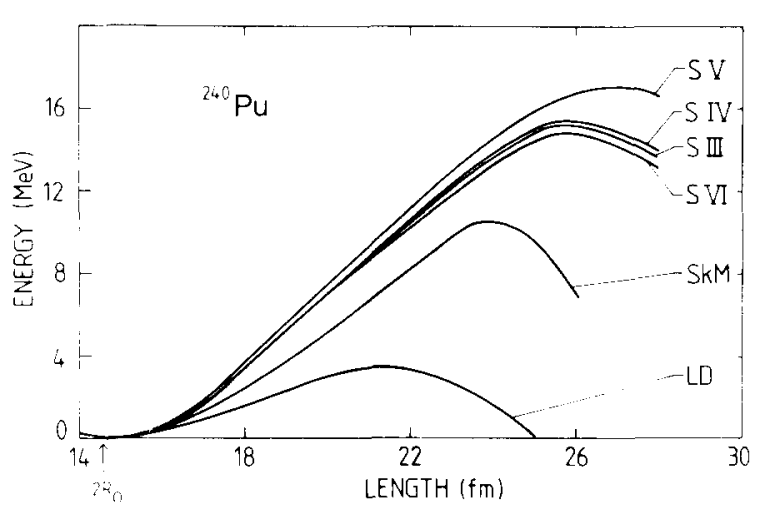

Fig. 2. Deformation energy curves for ${ }^{240} \mathrm{Pu}$ as in fig. 1 (with the $\{s, t, v\}$ shape parametrisation) tor five different Skyrme forces. The empirical LDM barrier is also shown.

though an even lower barrier was found there for the SkM force.

When comparing CHF results to the empirical barriers, one should keep in mind that the former contain spurious rotational energies due to the fact that Slater determinants are not eigenfunctions of the total angular momentum. To the extent that our semiclassical model is equivalent to an averaged (HF) system of independent particles (which is actually demonstrated here; see also refs. $[8,11])$, the bulk part of this spurious rotational energy is also contained in our results for $E_{\mathrm{ETF}}$. It can be estimated [3-5] to contribute $\approx 4-6 \mathrm{MeV}$ to the height of the average fission barriers. If this amount is deducted from our results shown in fig. 2, the barrier obtained with the force $\mathrm{SkM}$ is actually quite reasonable. As to the spurious centre of mass energies also contained in HF results, they should contribute less than $\approx 1 \mathrm{MeV}$ to the deformation energies if the traditional estimates are used $[1,3]$.

Before concluding this paper, we want to demonstrate the reliability of our semiclassical method by presenting in table 1 some results obtained with the force S III for a series of nuclei, including a hypothetical superheavy nucleus with $N=228, Z=126$. The first two columns contain the exact $[1,18]$ and selfconsistently Strutinsky-averaged [11] $\mathrm{HF}$ energies $E_{\mathrm{HF}}$ and $\widetilde{E}_{\mathrm{HF}}$, respectively ${ }^{\neq 1}$ (constrained to spherical shape for

\$1 No cm energy corrections and no pairing correlations are included. 
Table 1

Total energies in $\mathrm{MeV}, \mathrm{rms}$ radii in $\mathrm{fm}$ and diffusivities in $\mathrm{fm}$, obtained for spherical nuclei with the force S III. HF results are given along with those of our present investigation (denoted by ETF). $\widetilde{E}_{\mathrm{HF}}$ is the selfconsistently averaged HF energy [11].

\begin{tabular}{|c|c|c|c|c|c|c|c|c|}
\hline Nucleus & $E_{\mathrm{HF}}$ & $\widetilde{E}_{\mathrm{HF}}$ & $E_{\mathrm{ETF}}$ & $\left(r_{\mathrm{p}}\right)_{\mathrm{HF}}$ & $\left(r_{\mathrm{p}}\right)_{\mathrm{ETF}}$ & $\left(r_{\mathrm{n}}-r_{\mathrm{p}}\right)_{\mathrm{HF}}$ & $\left(r_{\mathrm{n}}-r_{\mathrm{p}}\right)_{\mathrm{ETF}}$ & $\left(a_{\mathrm{n}}-a_{\mathrm{p}}\right)_{\mathrm{ETF}}$ \\
\hline${ }_{20}^{40} \mathrm{Ca}$ & -325 & -322 & -327 & 3.41 & 3.39 & -0.04 & -0.04 & -0.010 \\
\hline${ }_{70}^{168} \mathrm{Yb}$ & -1312 & -1334 & -1356 & 5.20 & 5.19 & 0.08 & 0.09 & 0.065 \\
\hline${ }_{82}^{208} \mathrm{~Pb}$ & -1619 & -1598 & -1618 & 5.52 & 5.55 & 0.13 & 0.12 & 0.067 \\
\hline${ }_{94}^{240} \mathrm{Pu}$ & -1771 & -1784 & -1808 & 5.83 & 5.81 & 0.11 & 0.13 & 0.078 \\
\hline $\begin{array}{l}354 \\
126\end{array}$ & -2358 & -2348 & -2374 & 6.59 & 6.57 & 0.16 & 0.19 & 0.129 \\
\hline
\end{tabular}

${ }^{168} \mathrm{Yb}$ and ${ }^{240} \mathrm{Pu}$ in the HF case). Our semiclassical energies $E_{\mathrm{ETF}}$ given in column 3 agree quite well with the values $E_{\mathrm{HF}}$, being lower by $\approx 1-1.5 \%$. This slight overbinding might be due to some minor defects of the density functionals $\tau[\rho]$ and $J[\rho]$ used. We presently study the possibility to correct for it in a perturbative way, as it has been done in ref. [17].

Columns 4 and 5 in table 1 show the proton rms radii $r_{\mathrm{p}}$ obtained in $\mathrm{HF}[1,18]$ and our semiclassical calculations. The agreement within $\lesssim 0.5 \%$ is very satisfactory. Our model even reproduces rather accurately the neutron-proton difference in the rms radii, $r_{n}-r_{p}$, as seen in columns 6 and 7 . In the last column we show the surface thickness difference $a_{\mathrm{n}}-a_{\mathrm{p}}$ obtained in our calculations. It is interesting that it becomes larger with increasing neutron excess. For ${ }^{40} \mathrm{Ca}$, the protons are slightly more diffuse than the neutrons due to the Coulomb repulsion. Over the whole region considered, $a_{\mathrm{p}}$ is constant within a few percent $(\approx 0.35 \mathrm{fm})$, whereas $a_{\mathrm{n}}$ increases from $0.35 \mathrm{fm}\left({ }^{40} \mathrm{Ca}\right)$ to $0.47 \mathrm{fm}\left({ }^{354} \mathrm{Xx}\right)$, thus by $\approx 30 \%$. Since we found furthermore that the ratio $\rho_{0 \mathrm{n}} / \rho_{0 \mathrm{p}}$ stays almost constant $(\approx 0.97 \mathrm{~N} / \mathrm{Z})$ for all nuclei considered, we can conclude that the increase in $r_{n}-r_{p}$ observed is intimately connected with the increasing neutron diffuseness, as is evident from the strong correlation between the numbers in the last two columns of table 1 .

We note that the radial form of our densities eq. (1) are different from Fermi functions in the spherical case where $l_{\mathrm{q}}=\frac{1}{2}\left(r^{2}-R_{\mathrm{q}}^{2}\right)$, see ref. [14]. This explains the value of $a_{\mathrm{p}} \approx 0.35 \mathrm{fm}$ which seems much lower than the experimental number $0.55 \mathrm{fm}$. Indeed, fitting our densities by Fermi functions $a_{\mathrm{p}}$ would come close to $0.5 \mathrm{fm}$. We presently investigate the full variation of the densities by solving the Euler-Lagrange equations in order to see whether our parametrisation of the den. sities can be significantly improved.

In conclusion we remark that our semiclassical method reproduces rather accurately the averaged HF results. The use of the full functionals $\tau[\rho]$ and $J[\rho][9]$ up to fourth order not only leads to excellent binding energies and radii of spherical nuclei; particularly it also permits one to obtain reliable average deformation energies (within $\approx 2 \mathrm{MeV}$ ) in a very economical way.

It would be premature to draw definite conclusions about the suitability of different Skyrme forces to yield correct fission barriers, although the force SkM seems to be the favourite candidate of the cases shown in fig. 2. Before that, a detailed study of the role of their LD parameters such as surface and asymmetry coefficients, effective mass and incompressibility, is necessary. Such a study is now in progress and will be published along with some more details of our calculations.

We have greatly profited from the interest of B.K. Jennings in our investigations. We thank P. Quentin for providing us some of the HF results.

\section{References}

11] D. Vautherin and D. Brink, Phys. Rev. C5 (1972) 626; M. Beiner et al., Nucl. Phys. A238 (1975) 29.

[2] D. Gogny, in: Nuclear self-consistent fields, eds. G. Ripka and M. Porneuf (North-Holland, Amsterdam, 1975) p. 333; D. Gogny, Nucl. Phys. A237 (1975) 399.

[3] H. Flocard et al., in: Physics and chemistry of fission 1973, Vol. I (IAEA, Vienna, 1974) p. 221.

[4] J.F. Berger and M. Girod, in: Physics and chemistry of fission 1979, Vol. I (IAEA, Vienna, 1980) p. 265.

[5] M. Brack, in: Physics and chemistry of fission 1979, Vol. I (IAEA, Vienna, 1980) p. 227.

[6] P. Quentin and H. Flocard, Ann. Rev. Nucl. Part. Sci. 28 (1978) 523. 
[7] H. Krivine, J. Treiner and O. Bohigas, Nucl. Phys. A336 (1980) 155 .

[8] M. Brack, B.K. Jennings and Y.H. Chu, Phys. Lett. 65B (1976) 1.

[9] B. Grammaticos and A. Voros, Ann. Phys. (NY) 123 (1979) 359, part I: and part II, to be published.

[10] C. Guet, R. Bengtsson and M. Brack, in: Physics and chemistry of fission 1979, Vol. II (IAEA, Vienna, 1980), to be published; paper IAEA-SM/241-H3.

[11] M. Brack and P. Quentin, submitted to Nucl. Phys. for the S.G. Nilsson Memorial Volume; see also Phys. Lett. 56B (1975) 421.
[12] C. Guet and M. Brack, Z. Phys., to be published (Regensburg preprint 1980 ).

[13] J. Damgaard et al., Nucl. Phys. A 135 (1969) 432.

[14] M. Brack et al., Rev. Mod. Phys. 44 (1972) 320.

[15] W.D. Myers and W.J. Swiatecki, Nucl. Phys. 81 (1966) 1.

[16] A.K. Dutta and M. Kohno, McMaster Univ. preprint (1980).

[17] Y.H. Chu, B.K. Jennings and M. Brack, Phys. Lett. 68B (1977) 407.

[18] P. Quentin, Thèse d'Etat, Université de Paris-Sud, Orsay (1975), unpublished; and private communication (1980). 